\title{
Ambient intelligence for ubiquitous health and wellbeing
}

\author{
Mounir Mokhtari • Mohamed Ali Feki • \\ Chris D. Nugent
}

(C) Institut Télécom and Springer-Verlag 2010

The effects of age-related decline prevent many people from enjoying full, independent, productive, and highquality lives. Smart Homes have been proposed as an innovative solution in an attempt to address these issues in an effort to provide support to both elderly and people with disabilities in their daily life. In addition, Smart Homes technologies (ubiquitous computing) can be used as means to improve both the quality of care and wellbeing of dependent people. In one hand, ubiquitous health-based applications involving monitoring and processing of vital signs and enabling a rapid intervention in case of emergency are expected to become crucial services. In other hand, assistive services could provide a strong impact on the wellbeing of people. With such an approach, two main issues may be addressed: first, the person can remain within their own home and be safe and secure for an extended period of time, and second,

\section{Mokhtari ( $\square)$}

CNRS IPAL (UMI 2955) Singapore, Institut Telecom France, Institute for Infocomm Research (I2R/A-STAR),

1 Fusionopolis Way, \# 21-01 Connexis (South Tower),

Singapore 138632, Singapore

e-mail: Mounir.Mokhtari@it-sudparis.eu

\section{Ali Feki}

Senior Application Researcher Bell Labs,

Alcatel-Lucent Bell N.V,

Copernicuslaan 50 ,

2018 Antwerp, Belgium

e-mail: Mohamed_Ali.Feki@alcatel-lucent.com

\section{D. Nugent}

School of Computing and Mathematics, Faculty of Computing and Engineering, University of Ulster at Jordanstown,

Shore Road, Newtownabbey, Co,

Antrim BT37 0QB Northern Ireland, UK

e-mail: CD.Nugent@ulster.ac.uk offer a reformed approach for the way in which healthcare is delivered and managed. Embedded within these visions of creating a smart environment within which people can live for an extended period of time emerges the notion of ambient intelligent technologies. These technologies offer promising prospects to assist people with special needs. Nevertheless, they are an emerging research field which faces numerous challenges.

Ambient Assistive Living (AAL), which is a European initiative, deals with a new vision of the digital world, where computing, devices, and sensors are deployed everywhere (for example, at home, in buildings, and within urban spaces) to assist people. These technologies enable more intelligent and unobtrusive user interaction with the surrounding environment. The AAL community has the goal of including more intelligence within such environments. Such an increased level of intelligence has the potential to provide improved levels of user support in addition to helping users access the knowledge required to offer better decisions when interacting with these environments.

With the rapidly growing aging population on a global scale, the need of improving elderly wellbeing is getting crucial. Ambient Intelligence solutions are, therefore, increasingly becoming viable options for independent living. There is a need for increased sophistication in the homes and living environments of the elderly, and in the devices and artifacts that surround these living environments. The focus has moved beyond devices and electronics to reasoning, learning, monitoring, tracking, and providing context aware assistance, all within a framework of building accurate models covering the application domain. Despite the complexity and sophistication of this new generation of smart space, their realization must be intuitive (by providing innovative 
interaction models), intelligent (in the sense of understanding and predicting the needs of their users), flexible (in term of configurability of systems), and scalable for the management of large numbers of aging people and people with disabilities.

With this in mind, it has been the aim of this special issue of Annals of Telecommunications to provide a consolidated collection of recent Research and Development deployment case studies at an international level. This issue is comprised of a total of eleven papers, two of which represent a valuable presentation of the state-ofthe-art in the domain of healthcare issues and ICT solutions with the remaining nine papers presenting a detailed insight into technological solutions for real case studies. All papers are at the forefront of the exciting field of ambient intelligence in smart homes for the elderly and together present an excellent collection of ideas for perusal and further investigation.

The issue begins with a paper by Jeffrey Soar, from Australia, that describes the potential of information and communication technologies to support aging and independent living. The examples are taken from a real-world Smart Home environment and indicate the richness and flexibility of the modeling tools necessary to address the concerns of the next generation smart home. Tackling the specific population of people with Cognitive diseases, Nicola Armstrong, Chris Nugent, George Moore, and Dewar Finlay present an interesting assessment of the use of smart phones as a means of assistive technology for persons with Alzheimer's disease.

As a bridge paper toward deeply technical discussions, Mathieu Bouet and Guy Pujolle present their vision on a specific emerging technology: RFID in eHealth systems: They highlight a set of real applications with their respective challenges and perspectives.

Jit Biswas, Andrei Tolstikov, Maniyeri Jayachandran, Victor Foo, Aung Aung Phyo Wai, Clifton Phua, Weimin Huang, Louis Shue, Kavitha Gopalakrishnan, Jer-En Lee, and Philip Yap stress the focus on wearable and ambient sensors as an infrastructure layer enabling a middleware design for Health and wellness monitoring. Biswas and his colleagues underline a set of exemples from home-based care of elderly with mild dementia with a detailed description of their successful real deployments in hospital and Nursing Home environments in Singapore.

Fulvio Mastrogiovanni, Antonio Sgorbissa, and Renato Zaccaria introduce a novel behavioral framework for cognitive behavioral modeling, reasoning and assistance based on a highly developed logical theory of actions that go beyond data-centric behavioral models in that they govern an inhabitant's behavior by reasoning about its knowledge, actions, and environmental events. The paper proposes system architecture to illustrate the use of the proposed framework in facilitating assistive living.

In the same line of behavior tracking, Rachid Kadouche, Belkacem Chikhaoui, and Bessam Abdulrazak, from Canada, present user's behavior study for smart houses occupant prediction. This paper stresses the focus on learning user's habits to aid with their prediction in the future and serves as a user identification solution.

One area of consideration which emerges frequently when considering systems for eldercare is user-driven application creation. The paper by Carlos Baladron, Javier M. Aguiar, Javier Gobernado, Belén Carro, and Antonio Sánchez provides a detailed assessment of the entire issue of user involvement in home care application creation. The paper proposes a web based interface enabling a wide range of stakeholders to create home care applications by a means of simple template configuration and drag and drop user interface components. The authors validate their developed system through pilot test experimentation.

Michael P. Poland, Chris D. Nugent, Hui Wang, and Liming Chen present details of a study which aimed to profile behavioral patterns within smart environments based on spatial-frequency statistics. The work describes the approach adapted to process video signals collected from a number of experiments conducted within a smart kitchen environment. The long-term overarching goal of this work is to determine automatic approaches which can determine the optimal placement of sensors based on behavioral patterns.

Zdenka Uhríková, Chris D. Nugent, David Craig and Václav Hlaváč also investigate the usage of video based information within smart environments. Within their work, they present an innovative approach to detect repetitive behaviors of persons suffering from a form of dementia.

As a framework paper, Stéphane Renouard and Mounir Mokhtari propose a model and development Kit for a new Human Environment Interaction. The system is an intuitive and multimodal easy tool for caregivers and home care ecosystem actors to make the personalization of the smart behavior embedded into the environment reflects the user control.

The final paper proposed by Donna Hudson and Maurice E. Cohen investigates methods that can contribute to the delivery of home healthcare in the area of monitoring and support using intelligent agent methodologies. This paper is dealing with both people with dementia as well as remote monitoring of cardiac conditions.

Finally, the editors of this issue would like to thank the Editor-in-chief and Editorial Board of Annals of Telecom for providing them with opportunity to compile this edition. In addition, the editors are grateful to the authors and effort of the reviewers during the pier review process of all submitted papers. 